\title{
Recursos terapêuticos para pacientes com sintomas leves da COVID-19*
}

\author{
Therapeutic strategies for patients with mild symptoms of COVID-19*.
}

\author{
SARAIVA, Ana Carolina Lustosa ${ }^{1}$; BOMFIM, Ivo Saturno²; ALCANFOR, Thiago \\ Alexandre da Fonseca ${ }^{3}$; FURLANETTO, Karina Couto ${ }^{4}$; em nome do Comitê COVID-19 \\ da ASSOBRAFIR.
}

\begin{abstract}
Resumo
Este documento tem como objetivo trazer esclarecimentos sobre a atuação do fisioterapeuta em casos leves da COVID-19 e orientar acerca dos possíveis recursos que podem ser empregados no tratamento destes pacientes. O fisioterapeuta deve evitar contatos desnecessários com os pacientes. A real necessidade de atendimento presencial ou à distância deve ser avaliada considerando as individualidades de cada paciente, como sintomas respiratórios, comorbidades associadas e risco de evoluir com limitação funcional. Exercícios físicos terapêuticos estão indicados somente quando a condição do paciente permitir e limitam-se a uma intensidade baixa. Além disso, a terapia de remoção de secreção deve ser empregada apenas em pacientes hipersecretivos com incapacidade de tosse e expectoração. Atenção especial à segurança profissional e ao controle de transmissão devem ser empregada. Caso exista indicação de acompanhamento fisioterapêutico, o telemonitoramento pode ser considerado visando reduzir o risco de transmissão da COVID-19.
\end{abstract}

Palavras-chave: Fisioterapia; Infecções por coronavírus; Técnicas de Fisioterapia.

\footnotetext{
* Revisado por membros do Comitê COVID-19 da ASSOBRAFIR, nomeado por meio do memorando Nº03/2020. Esta publicação é uma atualização da Comunicação Oficial "Recursos terapêuticos para pacientes com sintomas leves da COVID-19", chancelada pelo Comitê COVID-19 da ASSOBRAFIR, originalmente escrita pelos mesmos autores e divulgada em 04/06/2020 no endereço eletrônico https://assobrafir.com.br/wp-content/uploads/2020/06/ ASSOBRAFIR COVID-19 Formas-Leves 2020.06.03.pdf.

${ }^{1}$ Departamento de Fisioterapia, Centro Universitário Vale do Salgado (UNIVS), Icó, Ceará. Hospital Regional do Cariri, Juazeiro do Norte, Ceará. Email: anacarolinalustosa@yahoo.com.br ACLS - https://orcid.org/0000-0002-9843-4952

${ }^{2}$ Hospital Regional do Cariri, Juazeiro do Norte, Ceará. Departamento de Fisioterapia, Centro Universitário Leão Sampaio (UNILEÃO), Juazeiro do Norte, Ceará. ISB - https://orcid.org/0000-0002-5875-9445

${ }^{3}$ Hospital Regional do Cariri, Juazeiro do Norte, Ceará. TAFA - https://orcid.org/0000-0001-6472-1825

${ }^{4}$ Programa de Pós-Graduação em Ciências da Reabilitação, Universidade Pitágoras-Unopar (UNOPAR), Londrina, Paraná, Brasil. Laboratório de Pesquisa em Fisioterapia Pulmonar (LFIP), Universidade Estadual de Londrina (UEL), Londrina, Paraná, Brasil. KCF - https://orcid.org/0000-0002-7496-7228
} 


\section{Abstract}

The current document aims to provide information about therapeuticstrategies that can be used by physiotherapists to treat patients with mild COVID-19. The physiotherapist must avoid unnecessary physical contact with these patients. The need for face-to-face or telehealth care should be analyzed according to characteristics of the patients such as their respiratory symptoms, comorbidities and potential risk of functional decline. Physical exercise is indicated whenever the patient's health condition allows, but low intensity exercise should be preferred. In addition, airway clearance techniques should only be used in patients with mucous hypersecretion who present with difficulties or inability to clear secretions independently. Special attention must be given to staff safety and contamination control. If there is indication for physiotherapy, telemonitoring may be considered to reduce the risk of contamination for COVID-19.

Keywords: Physiotherapy; Coronavirus infections; Physical Therapy Modalities.

\section{Objetivo}

O objetivo deste documento é trazer esclarecimentos sobre a necessidade de atuação do fisioterapeuta nos casos leves da infecção pelo novo coronavírus (COVID-19), bem como orientar acerca dos possíveis recursos que poderiam ser empregados no tratamento desses pacientes.

\section{Quadro clínico leve da COVID-19}

Apesar do novo coronavírus (SARS-CoV-2) apresentar alta transmissibilidade e capacidade para provocar casos de insuficiência respiratória grave, a maioria das ocorrências são de forma leve (cerca de 80\%). Estes pacientes normalmente não são hospitalizados e devem receber atenção especial dos serviços de atenção primária à saúde (APS), utilizando-se de estratégias de suporte e bem-estar, isolamento domiciliar e monitoramento ${ }^{1}$.

De acordo com a Organização Mundial de Saúde (OMS), os casos leves da COVID-19 podem ser interpretados como uma infecção não complicada que envolve o trato respiratório superior, expressa por sintomas inespecíficos como febre, fadiga, tosse (com produção ou não de secreções), inapetência, mal-estar, miastenia, dispneia, congestão nasal, anosmia, dor na garganta e cefaléia ${ }^{2}$. Mais raramente, podem surgir sintomas como náuseas, vômitos e diarreia. Entretanto, idosos e pacientes imunocomprometidos podem apresentar quadro clínico menos característicos ${ }^{2}$.

\section{Como agir frente aos pacientes com COVID-19 com quadro clínico leve?}

Nos pacientes suspeitos ou confirmados com COVID-19, em quaisquer estágios de gravidade da doença, o isolamento e outras estratégias de prevenção e controle de infecções são indicadas para conter e reduzir a transmissão do vírus ${ }^{2,3}$. De modo geral, o manejo clínico dos pacientes com quadro clínico leve, além do isolamento domiciliar por 14 dias após o início dos sintomas, consiste de medidas não farmacológicas como repouso, hidratação e alimentação adequada1. Deve-se priorizar o isolamento de idosos, gestantes, crianças e dos grupos de risco. Outra opção é o isolamento em hotéis, estádios ou ginásios, permanecendo nestes locais até que se afaste a possibilidade de infecção pelo novo coronavírus (14 dias de início dos sintomas, resolução da febre por mais de 72 horas sem antitérmicos e melhora dos sintomas respiratórios $)^{4}$. 
Mesmo em isolamento, estes pacientes precisam estar sob vigilância ativa e contínua, sendo indicado o acompanhamento do quadro a cada 48 horas, ou sempre que necessário, preferencialmente à distância, sendo a consulta presencial indicada somente quando o exame físico for necessário ${ }^{2}$. Uma vez isolados, uma alternativa para a assistência a esses pacientes pode incluir a teleconsulta e/ ou telemonitoriamento, conforme resolução 516/2020 do Conselho Federal de Fisioterapia e Terapia Ocupacional ${ }^{5}$.

Em razão das poucas evidências ainda disponíveis e dos riscos que envolvem a execução de procedimentos de fisioterapia nesta população, vale salientar que a atuação dos fisioterapeutas dependerá da fase da doença na qual o paciente se encontra, assim como os sintomas que possa apresentar. De maneira geral, as metas da fisioterapia no contexto da COVID-19 estão diretamente relacionadas à melhora da sensação de dispneia, manutenção da função pulmonar, prevenção de complicações osteomioarticulares, vasculares e respiratórias, prevenção e melhora de disfunções/ incapacidades, visando reduzir os efeitos deletérios do imobilismo, assim como a manutenção ou melhora da qualidade de vida e redução dos níveis de ansiedade e depressão ${ }^{6}$.

O fisioterapeuta deve identificar em cada paciente quais são as particularidades que indicam ou não a realização de intervenções, assim como determinar qual será o tipo de tratamento prescrito. Em alguns casos, o fisioterapeuta poderá realizar acompanhamento, monitorização ou orientações à distância, garantindo sempre a segurança do paciente, por exemplo, por meio do telemonitoramento, como mencionado anteriormente. Em outros casos, quando houver doenças associadas, a intervenção poderá ser presencial, e quando esta for indicada, sempre que possível, as atividades devem ser realizadas em ambientes arejados ${ }^{7}$. De modo geral, o fisioterapeuta deve evitar contato desnecessário com o paciente devido ao risco de contágio. Enfatizamos, porém, que o fisioterapeuta em atendimento presencial deve estar sempre atento ao uso correto dos equipamentos de proteção individual (EPIs), como a utilização de máscara N95, em virtude de procedimentos que podem gerar aerossóis. Além disso, é preciso fortalecer os cuidados em relação às medidas para evitar contágio.

De um modo geral, objetiva-se prevenir os agravos musculo-esqueléticos e melhorar os sintomas respiratórios. Adicionalmente, a atuação junto a esses pacientes, idealmente, deve incluir suporte psicológico e educação sobre a doença ${ }^{8}$. A seguir, os recursos de fisioterapia para pacientes com COVID-19 leve serão discutidos separadamente em dois grandes grupos "Exercícios terapêuticos" e "Fisioterapia respiratória".

\section{Exercícios Terapêuticos}

Durante a fase de isolamento, estes pacientes naturalmente aumentam o tempo em que ficam na posição sentada ou deitada, o que pode contribuir de forma expressiva para maior intolerância ao exercício, redução de força muscular, prejuízo de tosse e expectoração e maior risco de trombose venosa profunda (TVP), especialmente em indivíduos que fazem parte do grupo de risco, como idosos e obesos ${ }^{9}$. Dessa maneira, a fisioterapia deve ser baseada em uma avaliação abrangente das condições de saúde do paciente e das medidas de gerenciamento.

É importante ressaltar que pacientes com COVID-19 frequentemente apresentam febre, que é um achado intrinsicamente ligado a um maior gasto energético, sendo a prática de exercício físico nessas condições não é recomendada ${ }^{10}$. Quando indicado, os protocolos de exercícios terapêuticos devem ser realizados em intensidade de até 3 METs (Metabolic Equivalent Task), ou que não induzam 
à sensação de dispneia maior que 3 na escala de Borg modificada (CR10). Evidências sugerem que pacientes com tempo de diagnóstico de COVID-19 menor ou igual a sete dias e/ou tempo entre o início dos sintomas e o surgimento de dispneia leve igual ou menor que três dias, apresentam chance de evolução rápida para estados mais graves ${ }^{11}$. Portanto, os pacientes devem ser encorajados a manterem-se funcionais, realizar exercícios simples ou de atividades de vida diária (AVD) para evitar permanecer muito tempo do dia em atividades e/ou posturas sedentárias ${ }^{3,12}$.

Como recomendações gerais, além dos exercícios aeróbios de baixa intensidade, deve-se incluir no tratamento fisioterapêutico destes pacientes exercícios de força muscular, equilíbrio, alongamentos e treino de AVDs, uma vez detectadas incapacidades na realização ${ }^{11}$. Enfatizamos que essas condutas somente deverão ser realizadas quando a condição clínica do doente permitir.

\section{Fisioterapia Respiratória}

Em se tratando das condutas respiratórias, de acordo com recomendações internacionais recentes, nos pacientes suspeitos ou confirmados com COVID-19 que apresentem sintomas leves e sem comprometimento respiratório importante, a terapia de remoção de secreções não está indicada ${ }^{3}$. De fato, na fase inicial da doença, a indicação para terapia de remoção de secreção é mínima, uma vez que esses pacientes parecem não apresentar hipersecreção pulmonar. Dessa forma, o fisioterapeuta deve estar atento a otimização da ventilação e oxigenação desses pacientes ${ }^{13}$. Além disso, tem sido demonstrado que alguns recursos e técnicas utilizados pelo fisioterapeuta apresentam grande potencial para a disseminação de gotículas e aerossóis, o que pode aumentar a transmissão dos microrganismos causadores das infecções respiratórias como o SARS-CoV-25. Portanto, ações/ técnicas que envolvam tosse só devem ser consideradas após análise criteriosa da relação risco/ benefício em concordância com a equipe multidisciplinar ${ }^{6}$.

Em pacientes com prejuízo de tosse e dificuldades de expectoração, recomenda-se o uso de técnicas de remoção de secreção. Apenas para pacientes hipersecretivos estão indicadas técnicas como o ciclo ativo da respiração e a terapia com pressão positiva expiratória. O huffing, exigido ao final da técnica de Ciclo Ativo da Respiração, deve ser feito seguindo os mesmos cuidados e recomendações da etiqueta de tosse durante terapia de remoção de secreção. A terapia de expansão pulmonar também está indicada para auxiliar na terapia de remoção de secreção, em virtude do aumento do volume corrente facilitar a expectoração, com exceção a pacientes com hiperinsuflação pulmonar, em que as técnicas de expansão são contraindicadas. Nestes casos, os pacientes devem usar máscaras cirúrgicas ou máscaras de tecido caseiras durante a realização das técnicas, além de todo o cuidado em relação ao uso correto dos EPIs ser essencial ${ }^{3,11}$. No caso de pacientes que possuem doença neuromuscular, pode-se utilizar a máquina da tosse, com todos os cuidados com a higienização do equipamento, bem como com a proteção individual, conforme sugerido nas recomendações da ASSOBRAFIR ${ }^{12}$.

Durante a fisioterapia respiratória deve-se praticar a etiqueta da tosse e higiene. Recomendase que o paciente vire a cabeça para o lado, tussa em lenço de papel descartável e é desejável que o fisioterapeuta mantenha distância maior ou igual a dois metros, fora da "zona de explosão" e do sentido da tosse e expectoração. O descarte do papel deve ser realizado em lixeira fechada, separada e a lavagem das mãos é imprescindível após esses procedimentos ${ }^{3,6}$.

Outro ponto importante a ser comentado é sobre o uso de dispositivos auxiliares de fisioterapia respiratória, tanto para terapia de expansão como para remoção de secreções. $\mathrm{O}$ uso 
destes instrumentos pode ser considerado como de alto risco para transmissão da doença, devido ao potencial de disseminação do vírus. Da mesma forma, as técnicas de drenagem de secreções e alterações do fluxo expiratório devem ter sua aplicação limitada pelos mesmos motivos ${ }^{6,14}$. Quando utilizados, a desinfecção desses dispositivos deve ser realizada seguindo as recomendações da OMS, ANVISA e CDC para desinfecção de artigos semi críticos, empregando, no mínimo, desinfecção de alto nível. Para isso, recomenda-se o uso de desinfetantes listados na RDC no 35 da ANVISA ${ }^{15,16,17 .}$

A Figura 1 apresenta um fluxograma que auxilia na tomada de decisões quanto a intervenção fisioterápica.

Figura 1 | Fluxograma para auxiliar fisioterapeutas na tomada de decisões quanto aos atendimentos dos pacientes com a forma leve de COVID-19.

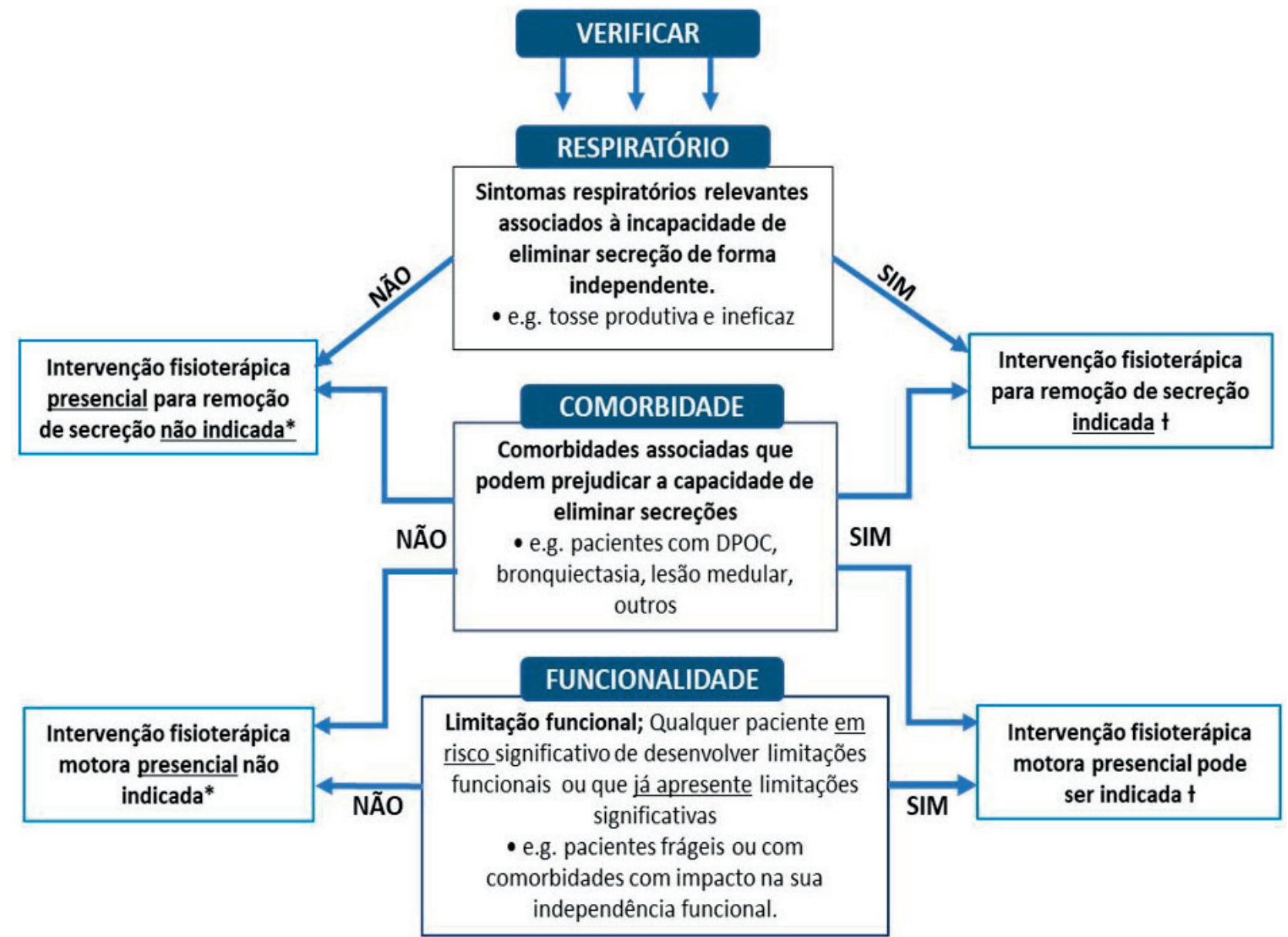

Legenda: *Objetivo: prevenir complicações futuras; sem indicação para fisioterapia presencial; o fisioterapeuta deve preferir a teleconsulta e/ou telemonitoramento se ainda julgar necessário; avaliação presencial deve ser realizada somente quando necessário realizar exame físico; indica-se o uso de cartilhas para orientação de exercícios (fisioterapia respiratória e motora). † Objetivo: será traçado individualmente; deve-se praticar etiqueta de tosse, usar lenço para expectoração que deve ser descartado imediatamente; o profissional em atendimento deve utilizar EPI e o paciente deve utilizar máscara sempre que possível; outros cuidados com procedimentos que possam gerar aerossóis devem ser tomados; o fisioterapeuta julgará a forma de oferta da intervenção (presencial ou telemonitoramento). 


\section{Considerações Finais}

A ASSOBRAFIR recomenda que o fisioterapeuta avalie a real necessidade de atendimento presencial ou à distância nos casos mais leves da COVID-19, considerando as individualidades de cada paciente, como sintomas respiratórios, comorbidades associadas e risco de evoluir com limitação funcional. Em pacientes com indicação para fisioterapia presencial, contato desnecessário deve ser evitado.

É importante ressaltar que em todo momento a segurança do profissional e o controle de transmissão e infecção devem ser considerados, e se necessário, recursos terapêuticos que seriam indicados em situações diferentes de uma pandemia, deverão ser evitados. Para isso, os gestores devem ter sabedoria ao considerar todos os fatores ambientais, pessoais, assim como a condição de saúde do paciente na tomada de decisões.

Por fim, tendo em vista a estimativa do Ministério da Saúde de crescimento dos casos de COVID-19 no Brasil, a atuação fisioterápica tem por objetivo prevenir que pacientes com a forma leve de COVID-19 e que já apresentavam limitações funcionais ou comorbidades prévias, evoluam com necessidade de internação hospitalar, o que ajuda a evitar o colapso dos serviços de saúde.

\section{Referências}

1. Brasil. Ministério da Saúde. Protocolo de manejo clínico do coronavírus (COVID-19) na atenção primaria à saúde [Internet]. Brasília: Secretaria de Atenção Primária à Saúde; 2020 Apr [cited 2020 Apr 15 2020]. Available from: https://portalarquivos.saude.gov.br/images/pdf/2020/April/08/20200408ProtocoloManejo-ver07.pdf

2. WHO. Clinical management of severe acute respiratory infection (SARI) when COVID-19 disease is suspected: interim guidance, 13 March 2020 [Internet]. Geneva: World Health Organization; 2020 [cited 2020 Mar 22]. Available from: https://apps.who.int/iris/handle/10665/331446

3. Thomas P, Baldwin C, Bissett B, Boden I, Gosselink R, Granger CL, et al. Physiotherapy management for COVID-19 in the acute hospital setting: clinical practice recommendations. J Physiother. 2020 Apr;66(2):73-82. doi: 10.1016/j.jphys.2020.03.011. Epub 2020 Mar 30.

4. WHO. Clinical management of severe acute respiratory infection (SARI) when COVID-19 disease is suspected: interim guidance [Internet]. Geneva: World Health Organization; 2020 [cited $2020 \mathrm{Mar}$ 21]. Available from: https://apps.who.int/iris/handle/10665/331446? show=full

5. COFFITO. Resolução no 516, 20 de março de 2020 - Teleconsulta, telemonitoramento e teleconsultoria [Internet]. Brasília: Conselho Federal de Fisioterapia e Terapia Ocupacional; 2020 [cited 2020 Apr 22]. Available from: https://www.coffito.gov.br/nsite/?p=15825

6. Arbillaga A, Pardàs M, Escudero R, Rodríguez R, Alcaraz V, Llanes S, et al. Fisioterapia respiratoria en el manejo del paciente con COVID-19: recomendaciones generales [Internet]. [place unknown]: Sociedad Española de Neumologia e Cirurgía Torácia; 2020 Mar 26 [cited 2020 Apr 14]. Available from: http://svmefr.com/wp-content/uploads/2020/03/COVID19-SEPAR-26 03 20.pdf

7. Yang F, Liu N, Wu JY, Hu LL, Su GS, Zheng NS. Pulmonary rehabilitation guidelines in the principle of $4 \mathrm{~S}$ for patients infected with 2019 novel coronavirus (2019-nCoV). Zhonghua Jie He He Hu Xi Za Zhi. 2020 Feb 5;43(0):E004. doi: 10.3760/cma.j.issn.1001-0939.2020.0004. Online ahead of print. Chinese. 
8. Chinese Association of Rehabilitation Medicine; Respiratory rehabilitation committee of Chinese Association of Rehabilitation Medicine; Cardiopulmonary rehabilitation Group of Chinese Society of Physical Medicine and Rehabilitation. Recommendations for respiratory rehabilitation of COVID-19 in adult. Zhonghua Jie He He Hu Xi Za Zhi. 2020 Apr 12;43(4):308-314. doi: 10.3760/ cma.j.cn112147-20200228-00206. Chinese.

9. Freitas APdaRF, Coester A, Schubert DUC, Guimarães HP. Protocolo suplementação de oxigênio em paciente com suspeita ou confirmação de infecção por COVID-19 [Internet]. São Paulo: Associação de Medicina Intensiva Brasileira; 2019 [cited 2020 Mar 22]. Available from: https://www. amib.org.br/fileadmin/user upload/protocolo oxigenioterapia covid19.pdf

10. Gosselink R, Bott J, Johnson M, Dean E, Nava S, Norrenberg M, et al. Physiotherapy for adult patients with critical illness: recommendations of the European Respiratory Society and European Society of Intensive Care Medicine Task Force on Physiotherapy for Critically Ill Patients. Intensive Care Med. 2008 Jul;34(7):1188-99. doi: 10.1007/s00134-008-1026-7. Epub 2008 Feb 19.

11. Grupo de Interesse em Fisioterapia Cardiorrespiratória. Fisioterapia respiratória em pessoas com COVID-19 [Internet]. [São Domingos de Rana]: Associação Portuguesa de Fisioterapeutas; 2020 May 4 [cited 2010 Mar 23]. Available from: https://www.gifcr-apf.com/

12. Furlanetto KC, Hernandes NA, Mesquita RB. Recursos e técnicas fisioterapêuticas que devem ser utilizadas com cautela ou evitadas em pacientes com COVID-19. Comunicação Oficial COVID-19. São Paulo: ASSOBRAFIR; 2020 [cited 2020 Apr 16]. Available from: https://assobrafir.com.br/covid19-recursos-e-tecnicas-que-devem-ser-utilizadas-com-cautela-ou-evitadas/

13. Moses, R. COVID 19: Respiratory Physioterapy on call information and Guidance. Version 2. London: The Chartered Society of Physiotherapy; 2020 Mar 17. Available from: https://www.csp.org. uk/documents/coronavirus-respiratory-physiotherapy-call-guidance

14. Indicazione per la Fisioterapia respiratoria in pazienti con infezione da COVID-19, aggiornato al 16/03/2020. Roma: Associazione Riabilitatori dell'insufficienza Respiratoria; 2020 [cited 2020 Mar 20]. Available from: https://aifi.net/emergenza-covid-19-indicazioni-per-fisioterapisti-e-pazienti/

15. Miranda AMFde, Quintini APN, Calicchio LG, Martins L, Silva MVDda, Bandeira OB, et al. Nota técnica: coronavírus e a CME [Internet]. São Paulo: NasceCME; 2020 [cited 2020 May 20]. Available from: http:// nascecme.com.br/2014/wp-content/uploads/2020/04/2a-Edição-Coronavírus-e-a-CME-C-21-04-20.pdf

16. United States. Department of Health \& Human Services. Center for Disease Control and Prevention. Infection Control. Disinfection and sterilization. Summary of Recommendations. HICPAC Indications for Sterilization, High-Level Disinfection, and Low-Level Disinfection. [Internet]. Atlanta, GA: Centers for Disease Control and Prevention (U.S.); 2020. Available from: https://www.cdc. gov/infectioncontrol/guidelines/disinfection/index.html

17. ANVISA. Resolução de Diretoria Colegiada no 35, 16 de agosto de 2010. Brasília: Agência Nacional de Vigilância Sanitária; 2020 [cited 2020 May 20]. Available from: http://portal.anvisa.gov. br/documents/10181/2718376/RDC 35 2010.pdf/823d5216-c173-4046-8a87-a3d297f99d87.

Submissão em: 08/06/2020

Aceito em: 15/07/2020 